\title{
Protective effects of combined treatment with mild hypothermia and edaravone against cerebral ischemia/reperfusion injury via oxidative stress and Nrf2 pathway regulation
}

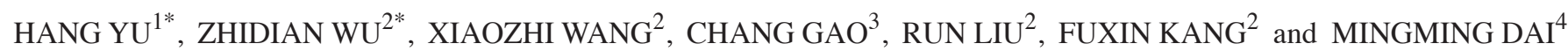 \\ ${ }^{1}$ Department of Critical Care Medicine, Hainan Medical University; ${ }^{2}$ Department of Critical Care Medicine, \\ The Second Affiliated Hospital of Hainan Medical University; ${ }^{3}$ Department of Pathophysiology, Hainan Medical University; \\ ${ }^{4}$ Department of Neurology, The Second Affiliated Hospital of Hainan Medical University, Haikou, Hainan 570311, P.R. China
}

Received November 5, 2019; Accepted April 27, 2020

DOI: $10.3892 /$ ijo.2020.5077

\begin{abstract}
Mild hypothermia (MH) and edaravone (EDA) exert neuroprotective effects against cerebral ischemia/reperfusion (I/R) injury through activation of the nuclear factor erythroid 2-related factor 2 (Nrf2) pathway. However, whether $\mathrm{MH}$ and EDA exert synergistic effects against cerebral I/R injury remains unknown. The aim of the present study was to investigate the effects and mechanism of action of $\mathrm{MH}$ in combination with EDA in cerebral I/R injury. A rat cerebral I/R injury model was constructed by middle cerebral artery occlusion (MCAO) followed by reperfusion, and the mice were treated by $\mathrm{MH}$, EDA or the inhibitor of the Nrf2 signaling pathway brusatol (Bru). It was observed that mice treated by MCAO had higher neurological deficit scores and oxidative stress levels, and low spatial learning and memory capacity; moreover, the CA1 region of the hippocampi of the mice exhibited reduced neuronal density and viability, and reduced mitochondrial dysfunction. However, $\mathrm{MH}$ in combination with EDA reversed the effects of MCAO, which were blocked by Bru injection. The levels of glutathione (GSH), GSH peroxidase, catalase and superoxide dismutase in rat ischemic hemisphere tissues were reduced by Bru. Western blotting demonstrated that the combined treatment with $\mathrm{MH}$ and EDA promoted the nuclear localization of $\mathrm{Nrf2}$, and increased the levels of $\mathrm{NAD}(\mathrm{P}) \mathrm{H}$ quinone oxidoreductase and heme oxygenase (HO)-1. In conclusion, $\mathrm{MH}$ combined with EDA
\end{abstract}

Correspondence to: Dr Mingming Dai, Department of Neurology, The Second Affiliated Hospital of Hainan Medical University, 48 Baishuitang Road, Haikou, Hainan 570311, P.R. China

E-mail: lming_mliu@163.com

${ }^{*}$ Contributed equally

Key words: mild hypothermia, edaravone, cerebral ischemia/reperfusion injury, nuclear factor erythroid 2-related factor 2 exerted synergistic neuroprotective effects against cerebral I/ R injury involving changes in the Nrf2/HO-1 pathway.

\section{Introduction}

Stroke, which is characterized by loss of neurological function caused by ischemia of the brain, intracerebral hemorrhage or subarachnoid hemorrhage (1), is associated with high morbidity and mortality rates $(2,3)$. It has been demonstrated that, by inducing excitotoxicity, cerebral ischemia/reperfusion (I/R) injury is a critical factor responsible for poor prognosis in patients with ischemic stroke. Stroke disrupts calcium ion homeostasis, causes overproduction of free radicals and inflammatory cytokines, and promotes cell apoptosis (4). Currently, although thrombolytic, endovascular and adjuvant novel therapies have been developed for stroke $(5,6)$, they have been proven insufficient in achieving the desired outcome. Therefore, a better understanding of the mechanisms underlying the development of cerebral $\mathrm{I} / \mathrm{R}$ injury is required.

Mild hypothermia (MH) exerts neuroprotective effects against cerebral ischemia. It was previously reported that $\mathrm{MH}$ reduces brain hemorrhage and blood-brain barrier disruption after stroke (7), and that it may alleviate cerebral ischemic injury in diabetic patients through promoting autophagy and inhibiting pyroptosis (8). Researchers also demonstrated that inhibition of Notch3 and Notch4 signaling is involved in the protective effect of $\mathrm{MH}$ against cerebral ischemic injury (9). Moreover, $\mathrm{MH}$ promotes long-term white matter integrity and inhibits neuroinflammation in mice with ischemic brain injury (10). These previous findings indicate that MH may be of therapeutic value in cerebral I/R injury.

Edaravone (EDA; 3-methyl-1-phenyl-2-pyrazolin-5-one) is a free radical scavenger. Evidence has indicated that EDA protects the brain against cerebral ischemic injury, and it may inhibit microglia-mediated neuroinflammation in rats with cerebral ischemic injury (11). It has also been demonstrated that EDA protects neuronal cells from ischemic injury by inhibiting the translocation of 5-lipoxygenase to the nuclear membrane, thereby blocking the 5-lipoxygenase signaling pathway (12). Moreover, EDA combined with MH significantly improves neuroprotection in rats exposed to hypoxia (13). 
Thus, it was inferred that EDA in combination with MH may exert a synergistic effect against cerebral I/R injury.

The transcription factor nuclear factor erythroid 2-related factor $2(\mathrm{Nrf} 2)$ is a central modulator in multiple biochemical processes, such as redox, protein and metabolic homeostasis. Nrf2-based therapeutics have been developed for treating various cardiovascular, kidney and liver diseases (14). It was previously demonstrated that EDA protects the nervous system from toxicity through activating the Nrf2 signaling pathway (15). However, whether the positive effects of EDA on cerebral I/R injury are mediated through the activation of the Nrf2 signaling pathway remains unclear.

Therefore, in the present study, a cerebral I/R model in rats was constructed to explore the potential synergistic effects and the mechanism underlying the combination of EDA with $\mathrm{MH}$ in I/R injury. In addition, brusatol (Bru), an inhibitor of the Nrf2 signaling pathway, was also used to investigate the effects of Nrf2 signaling on I/R injury.

\section{Materials and methods}

MCAO model and drug treatment. A total of 60 healthy adult Sprague-Dawley male rats, 9-10 weeks old and weighing 300-320 g, were purchased from Vital River Laboratories Co., Ltd. All the animals were housed under specific pathogen-free conditions with a 12-h dark/light cycle at $25^{\circ} \mathrm{C}$, and fed standard food and aseptic water. All the experiments were approved by the Institutional Animal Ethics Committee of Hainan Medical University (approval no. C2017051922A).

Focal I/R in each rat was created by middle cerebral artery occlusion (MCAO). Briefly, the rats were anesthetized by $3 \%$ isoflurane at $50 \mathrm{mg} / \mathrm{kg}$ body weight (1235809; Sigma-Aldrich; Merck KGaA) administered by intraperitoneal injection, while monitoring the heart rate. Then, the end of the common cerebral artery (CCA) nearer to the heart was closed by a nylon suture. A special nylon suture with a spherical end (diameter $0.18 \mathrm{~mm}$ ) was inserted into the brain of each rat following the direction of the CCA and stopped when the thread entered the middle cerebral artery (MCA). After occlusion for $120 \mathrm{~min}$, the thread was dismantled to allow reperfusion for $24 \mathrm{~h}$. The rats treated by $\mathrm{MCAO}$ were then randomly divided into 5 groups as follows: MCAO, MCAO + MH, MCAO + EDA, $\mathrm{MCAO}+\mathrm{MH}+\mathrm{EDA}$ and $\mathrm{MCAO}+\mathrm{MH}+\mathrm{EDA}+\mathrm{Bru}$ groups ( $n=10$ rats per group). Furthermore, 10 rats in the sham group were treated in a similar manner but without arterial occlusion. Immediately after MCAO, ice was placed under the skull of the rats treated by $\mathrm{MH}$, with the temperature of the temporalis muscle maintained at $32-34^{\circ} \mathrm{C}$ for $2 \mathrm{~h}$. EDA (M70800; Sigma-Aldrich; Merck KGaA) and Bru (SML1868, Sigma-Aldrich; Merck KGaA) were diluted in physiological saline $(16,17)$, and $3.0 \mathrm{mg} / \mathrm{kg}$ EDA or $1.0 \mathrm{mg} / \mathrm{kg}$ Bru were immediately intraperitoneally injected into the rats after MCAO. No rats died during the experiments. The rats were euthanized by $3 \%$ isoflurane $(150 \mathrm{mg} / \mathrm{kg}$ body weight) through intraperitoneal injection $24 \mathrm{~h}$ later and, after cardiac arrest was confirmed, their brain tissues were collected for the different assays.

Neurological deficit score. The modified Longa's Scoring System (18) was used to evaluate the neurological deficits of the rats at $24 \mathrm{~h}$ after reperfusion as follows: 0 , no deficit; 1 , inability to stretch the contralateral forelimb fully; 2 , circling to the contralateral side; 3 , falling over to the contralateral side; and 4, no spontaneous locomotor activity. A higher score indicated a more severe neurological deficit.

Brain 2,3,5-triphenyltetrazolium chloride (TTC) staining and infarct size calculation. The rats were euthanized and their brain tissues were collected, cut into 3-mm sections, and placed in a water bath with $2 \%$ TTC solution for $30 \mathrm{~min}$ at $37^{\circ} \mathrm{C}$ in the dark. The stained sections were photographed and then analyzed by AlphaImager HP 1.0 software (Alpha Innotech Corp) to calculate the infarct area rates.

Immunofluorescence staining. Immunofluorescent staining of NeuN was conducted to determine the neuronal density in the ischemic hippocampi. Briefly, the rats in each group were subjected to deep anesthesia 3 days after MCAO. The brain tissues were isolated, exposed to $4 \%$ paraformaldehyde (PFA) at $4^{\circ} \mathrm{C}$ overnight and then cryoprotected in $30 \%$ sucrose solution at $4^{\circ} \mathrm{C}$ overnight. When tissues sank to the bottom, they were frozen in optimal cutting temperature compound, and $25-\mu \mathrm{m}$ brain sections were prepared using a Leica microtome (CM3050 S; Leica Microsystems GmbH). Next, anti-rat NeuN antibody (1:500; cat. no. PA5-78499, Thermo Fisher Scientific, Inc.) was incubated with free-floating sections. Subsequently, secondary antibody conjugated with Alexa Fluor ${ }^{\circledR}$ Plus 488 (cat. no. A32731, Thermo Fisher Scientific, Inc.) was then incubated with the sections at $25^{\circ} \mathrm{C}$ for $2 \mathrm{~h}$. The images were captured using a confocal laser scanning microscope (CLSM; LSM 510 META, Carl Zeiss AG), with five fields examined for each sample (magnification, x400). Neuronal changes were quantified against sham control or other groups.

Barnes maze task. Barnes maze task was performed to examine the spatial learning and memory capacity of the rats. Briefly, the tests were conducted 11 days after MCAO. During testing, the time that each rat spent on finding the hidden escape box was recorded as escape latency. At 12 days after MCAO, the escape box was removed, and the time that the rats spent on finding the target quadrant within $90 \mathrm{sec}$ was recorded as quadrant occupancy. The behavioral traces of each rat were recorded by a camera and analyzed by ANY-maze 4.8 video tracking software (Stoelting Co.).

MitoTracker Red staining. Mitochondrial membrane potential (MMP) was detected using MitoTracker Red staining, as previously described (12). Briefly, MitoTracker ${ }^{\circledR}$ Red CMXRos (50 ng/ml in $100 \mu \mathrm{l}$ saline; C1049, Beyotime Institute of Biotechnology) was injected via the tail vein into the rats 5 min before euthanasia. Then, the brain tissues were isolated and fixed in $4 \%$ PFA at $4^{\circ} \mathrm{C}$. Hippocampal tissue were cut into $25-\mu \mathrm{m}$ sections and stained by DAPI (D8417; Sigma-Aldrich; Merck $\mathrm{KGaA}$ ) at $37^{\circ} \mathrm{C}$ for $10 \mathrm{~min}$. Images were then captured using a Zeiss CLSM (Carl Zeiss AG), and analyzed by LSM 510 META imaging software, version 2.01 (Carl Zeiss AG).

Oxidative stress parameters. For detecting oxidative stress parameters in rat ischemic hemisphere tissues, lipid peroxidation assay kit (MAK085; Sigma-Aldrich; Merck KGaA), 
glutathione (GSH) assay kit (CS0260; Sigma-Aldrich; Merck $\mathrm{KGaA}$ ), GSH peroxidase (PX) assay kit (S0058, Beyotime Institute of Biotechnology), catalase (CAT; S0051, Beyotime Institute of Biotechnology) and superoxide dismutase (SOD; S0109, Beyotime Institute of Biotechnology) were purchased and performed according to the manufacturer's instructions. Briefly, the tissue $(\sim 10 \mathrm{mg})$ was homogenized on ice in $300 \mu \mathrm{l}$ cell lysis buffer (MCL1; Sigma-Aldrich; Merck KGaA). For the detection of malondialdehyde (MDA) content, insoluble materials and proteins were removed from homogenized tissues by centrifugation at $13,000 \mathrm{x}$ g at $4^{\circ} \mathrm{C}$ for $10 \mathrm{~min}$; $150 \mu$ l water containing $3 \mu$ l butylated hydroxytoluene (100X) and $2 \mathrm{~N}$ perchloric acid was then added to the tissues and vortexed at $25^{\circ} \mathrm{C}$. Next, $200 \mu 1$ supernatant was mixed with $600 \mu \mathrm{l}$ thiobarbituric acid solution and incubated at $95^{\circ} \mathrm{C}$ for $60 \mathrm{~min}$. Subsequently, $200 \mu 1$ reaction mixture were added into a 96-well plate and the absorbance at $532 \mathrm{~nm}$ was determined by a microplate reader (PLUS 384, Molecular Devices, LLC). For the detection of GSH, $5 \mu 1$ homogenized tissue was deproteinized using $5 \mu 15 \% 5$-sulfosalicylic acid solution. Subsequently, $10 \mu \mathrm{l}$ mixture was mixed with $150 \mu \mathrm{l}$ working solution consisting of $1 \mathrm{X}$ assay buffer, enzyme solution and DTNB, and incubated for $5 \mathrm{~min}$ at $25^{\circ} \mathrm{C}$. Next, $50 \mu \mathrm{l}$ NADPH $(0.16 \mathrm{mg} / \mathrm{ml})$ was added into the mixture at $25^{\circ} \mathrm{C}$, and the absorbance at $412 \mathrm{~nm}$ was detected. For the detection of GSH peroxidase (GSH-PX), $180 \mu \mathrm{l}$ GSH-PX buffer, $5 \mu \mathrm{l}$ of diluted sample, $11 \mu 1$ GSH-PX working solution and $4 \mu 1$ $15 \mathrm{mM}$ superoxide (Cum-OOH) were added into a 96-well plate and incubated for $20 \mathrm{~min}$ at $25^{\circ} \mathrm{C}$, and the absorbance at $340 \mathrm{~nm}$ was determined. For the detection of CAT, $10 \mu \mathrm{l}$ diluted sample were incubated with $10 \mu 1250 \mathrm{mM}$ hydrogen peroxide and $30 \mu \mathrm{l}$ detection buffer at $25^{\circ} \mathrm{C}$ for $5 \mathrm{~min}$, and then the absorbance at $520 \mathrm{~nm}$ was read. For the detection of SOD, $20 \mu 1$ diluted sample were incubated with $160 \mu 1$ NBT solution and $20 \mu \mathrm{l}$ reaction regent at $25^{\circ} \mathrm{C}$ for $30 \mathrm{~min}$, and the absorbance at $560 \mathrm{~nm}$ was read.

Mitochondrial dysfunction detection. Mitochondrial dysfunction in the ischemic hemisphere tissues of the rats was evaluated by detecting the activity of cytochrome c using a cytochrome c apoptosis assay kit (K257-100, BioVision, Inc.). The cytochrome $\mathrm{c}$ released from mitochondria was detected by western blotting using the cytochrome $\mathrm{c}$ antibody in the kit. The relative content of cytochrome $\mathrm{c}$ in each group was determined against that in the sham group. Caspase-3 enzyme activity in rat ischemic hemisphere tissues was measured using a commercial kit (CASP3C; Sigma-Aldrich; Merck $\mathrm{KGaA})$.

Western blotting. After treatment for $24 \mathrm{~h}$, the ischemic brain tissues were separated and homogenized on ice. Nuclear and cytoplasmic proteins were extracted using nuclear and cytoplasmic extraction kits (SC-003, Invent Biotechnologies, Inc.). Equal amounts of protein $(50 \mu \mathrm{g})$ were separated on 12\% SDS-PAGE and transferred onto PVDF membranes (EMD Millipore). After blocking the membranes for $1 \mathrm{~h}$ in $5 \%$ non-fat milk, the bands were exposed to primary antibodies against $\operatorname{Nrf} 2(1: 2,000$, cat. no. ab137550, Abcam), heme oxygenase-1 (HO-1; 1:2,000; cat. no. ab13243, Abcam), $\mathrm{NAD}(\mathrm{P}) \mathrm{H}$ quinone oxidoreductase (NQO1; 1:2,000, cat. no. ab28947, Abcam), $\beta$-actin (1:5,000, cat. no. ab8226, Abcam) and histone $\mathrm{H} 3\left(1: 5,000\right.$, cat. no. ab1791, Abcam) at $4^{\circ} \mathrm{C}$ overnight. Next, the bands were incubated with anti-rabbit $\operatorname{IgG}$ (cat. no. SA00001-2, ProteinTech Group, Inc.) or anti-mouse IgG (cat. no. SA00001-1, ProteinTech Group, Inc.) for $2 \mathrm{~h}$. The bands were finally developed by ECL (Pierce; Thermo Fisher Scientific, Inc.).

Primary culture of hippocampal neurons. Briefly, the CA1 region of the rat hippocampus was rapidly separated 3 days after MCAO and sectioned, and then exposed to trypsin for $30 \mathrm{~min}$ at $37^{\circ} \mathrm{C}$. After that, the digested cells were cultured in DMEM (11965084; Thermo Fisher Scientific, Inc.) with 10\% FBS (10100147; Gibco; Thermo Fisher Scientific, Inc.), then transferred to an incubator with $5 \% \mathrm{CO}_{2}$ at $37^{\circ} \mathrm{C}$ for $2 \mathrm{~h}$. The culture medium was replaced once every 2 days. After culture for $24 \mathrm{~h}$, hippocampal neurons were used for the subsequent experiments. The purity of neurons was determined by immunofluorescence staining of NeuN.

Cell viability. The viability of hippocampal neurons was measured by MTT assay. Briefly, the neurons $(2,000$ cells/well $)$ were cultured in 96-well culture plates for $24 \mathrm{~h}$. Then, MTT solution $(5 \mathrm{mg} / \mathrm{ml})$ was added into each well at $37^{\circ} \mathrm{C}$ for $4 \mathrm{~h}$. Next, MTT was removed, and $150 \mu \mathrm{l}$ DMSO were added into the wells to solubilize purple formazan crystals. Absorbance at $570 \mathrm{~nm}$ was then read using a microplate reader (PLUS 384, Molecular Devices, LLC).

Statistical analysis. Statistical data were analyzed using SPSS 19.0 (SPSS Inc.) and are shown as mean \pm standard deviation. Statistical differences were analyzed by one-way analysis of variance followed by Tukey's multiple comparisons test. $\mathrm{P}<0.05$ was considered to indicate statistically significant differences.

\section{Results}

Effects of MH, EDA and Bru on neuronal survival in the CAI region of the rat hippocampus after $M C A O$. The neurological deficits were evaluated by the five-point scale $24 \mathrm{~h}$ after I/R, and the results revealed that the neurological deficit score of the rats subjected to MCAO was higher compared with those in the sham group $(\mathrm{P}<0.001$, Fig. 1A). The neurological deficit scores of the rats treated by MH or EDA were reduced compared with those in the MCAO group ( $\mathrm{P}<0.01$, Fig. $1 \mathrm{~A})$. The neurological deficit score was the lowest in rats treated by MCAO, MH and EDA, whereas the protective effects of MH and EDA on the nervous system of rats injected with the Nrf2 pathway inhibitor Bru were significantly reduced $(\mathrm{P}<0.001$, Fig. 1). Furthermore, immunofluorescence staining and a neuronal cell marker, NeuN, were used to analyze neuronal survival in the CA1 region of the rat hippocampus after MCAO. As shown in Fig. 1B and C, the number of surviving neurons was reduced in the MCAO group, but was increased in the $\mathrm{MCAO}+\mathrm{MH}$ and MCAO + EDA groups (Fig. 1B and C). In addition, the rats co-treated with $\mathrm{MH}$ and EDA exhibited a more marked recovery of neuronal number after $I / R$, while Bru reversed the protective effects of MH and EDA $(\mathrm{P}<0.001$, Fig. 1B). 
A

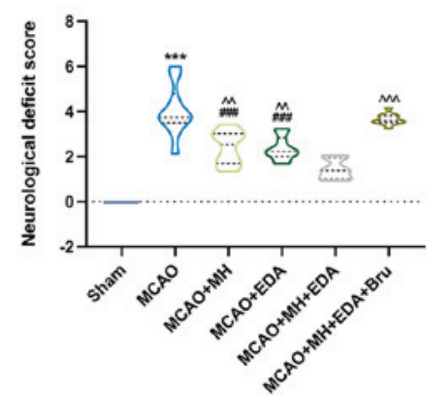

C

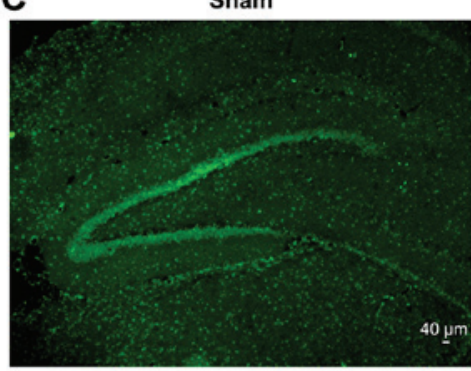

MCAO+EDA

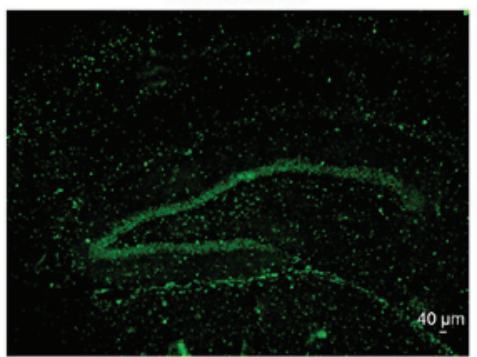

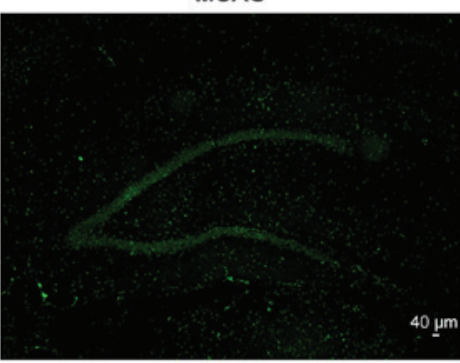

MCAO+MH+EDA

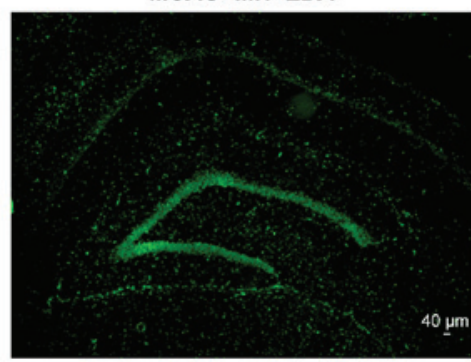

B

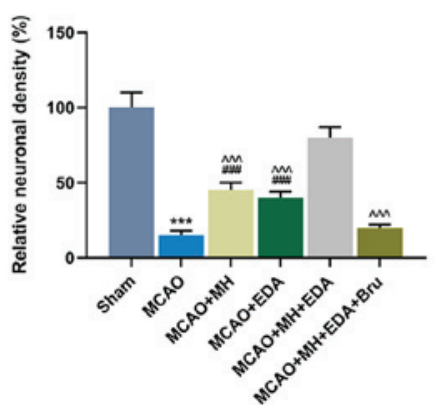

MCAO
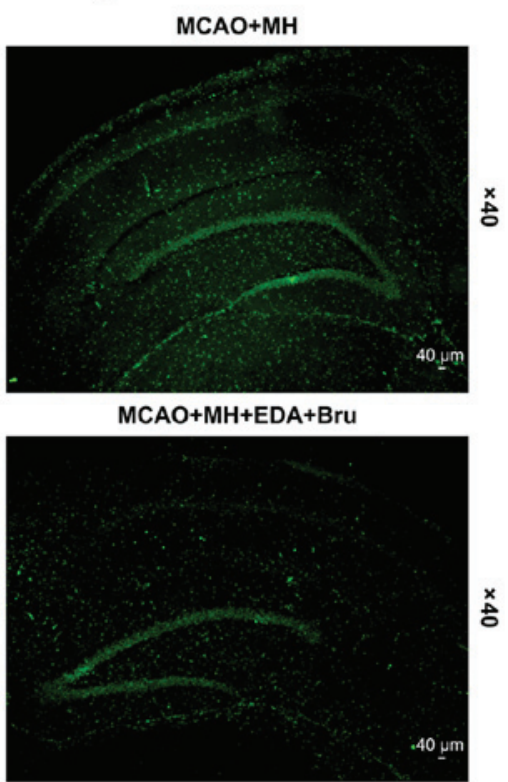

Figure 1. Effects of MH, EDA and Bru on neuronal survival in the CA1 region of the hippocampi after MCAO. (A) The neurological deficit scores of the rats in different groups were evaluated by a five-point scale $24 \mathrm{~h}$ after reperfusion. 10 mice were in each group. (B) Quantitative analyses of neuronal survival in the hippocampal CA1 pyramidal layer. The density of the surviving neurons was quantified as percentage changes against the sham control group. ${ }^{* * *} \mathrm{P}<0.001$ vs. sham. ${ }^{\# \# \#} \mathrm{P}<0.001$ vs. MCAO. ${ }^{\wedge} \mathrm{P}<0.01$ and ${ }^{\wedge \wedge} \mathrm{P}<0.001$ vs. $\mathrm{MCAO}+\mathrm{MH}+\mathrm{EDA}$. (C) Representative images of immunofluorescence labeling for NeuN in the ischemic hippocampus at day 3 after MCAO; $=10$ mice per group. Scale bar: $40 \mu \mathrm{m}$. Magnification, $\mathrm{x} 40$. MCAO, middle cerebral artery occlusion; MH, mild hypothermia; EDA, edaravone; Bru, brusatol.

Effects of $M H$, EDA and Bru on spatial learning, memory and mitochondrial function of the rats after MCAO treatment. The escape latency and quadrant occupancy were evaluated by performing the Barnes maze task to explore the effects of $\mathrm{MH}$, EDA and Bru on spatial learning and memory capacity of the rats after MCAO treatment. It was observed that the rats in the MCAO group spent a longer time on finding the hidden escape box (escape latency) compared with those in the sham group $(\mathrm{P}<0.001$, Fig. 2A), whereas treatment with $\mathrm{MH}$ or EDA alone, or combined treatment with $\mathrm{MH}$ and EDA, could significantly shorten the escape latency compared with rats in the MCAO group ( $\mathrm{P}<0.001$, Fig. 2A). In addition, Bru injection increased the escape latency in rats treated by $\mathrm{MH}$ and EDA $(\mathrm{P}<0.001$, Fig. 2A). The rats in the MCAO group spent a shorter time (quadrant occupancy) in the target quadrant compared with those in the sham group ( $\mathrm{P}<0.001$, Fig. 2B). However, $\mathrm{MH}$ and EDA increased the quadrant occupancy of the rats $(\mathrm{P}<0.001$, Fig. 2B), and Bru injection blocked the effect of co-treatment with $\mathrm{MH}$ and EDA $(\mathrm{P}<0.001$, Fig. 2B). MitoRed fluorescence staining was performed to detect the MMP, and the data indicated that MCAO reduced the fluorescence intensity of MMP, which indicated mitochondrial depolarization and collapse of the MMP, whereas MH and EDA increased the fluorescence intensity. Additionally, co-treatment with $\mathrm{MH}$ and EDA caused a more significant increase of fluorescence intensity compared with either treatment alone $(\mathrm{P}<0.001$, Fig. 2C and D), which, however, was reduced by Bru injection $(\mathrm{P}<0.001$, Fig. 2C and D).

Effects of MH, EDA and Bru on the levels of oxidative stress biomarkers, neuronal viability, cytotoxicity and caspase-3 activity. Oxidative stress is involved in cerebral I/R. Thus, oxidative stress was further assessed by detecting the levels of MDA, GSH, GSH-PX, CAT and SOD in ischemic hemisphere tissues (Fig. 3D and E). The results demonstrated that the level of MDA was increased in the MCAO group compared with that in the sham group, while $\mathrm{MH}$ or EDA treatment, or $\mathrm{MH}$ and EDA co-treatment, could partly reverse the increase in MDA content caused by MCAO ( $\mathrm{P}<0.001$, Fig. 3A). Additionally, Bru treatment reversed the effect of co-treatment with $\mathrm{MH}$ and EDA on MDA levels $(\mathrm{P}<0.001$, Fig. 3A). Furthermore, the levels of GSH, GSH-PX, CAT and SOD in rat ischemic hemisphere tissues were reduced by MCAO, but increased by $\mathrm{MH}$ and EDA treatment or co-treatment $(\mathrm{P}<0.001$, Fig. 3B-E). Additionally, the levels of GSH, GSH-PX, CAT and SOD in rat ischemic hemisphere tissues were reduced in 
A

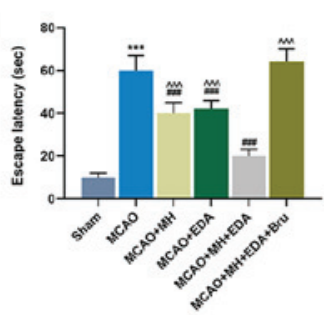

B

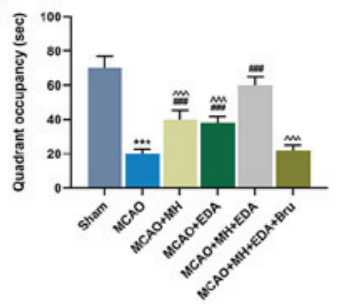

C

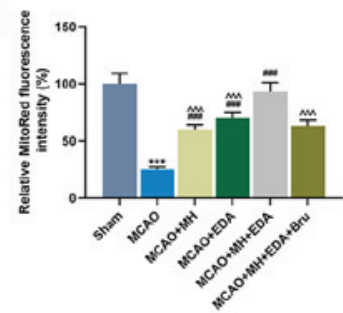

D

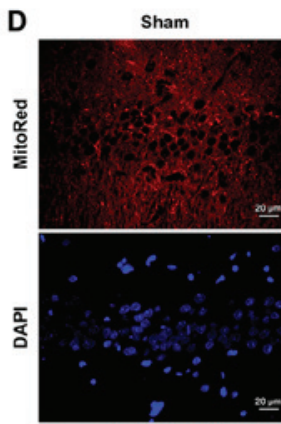

MCAO

MCAO+MH

$M C A O+E D A$

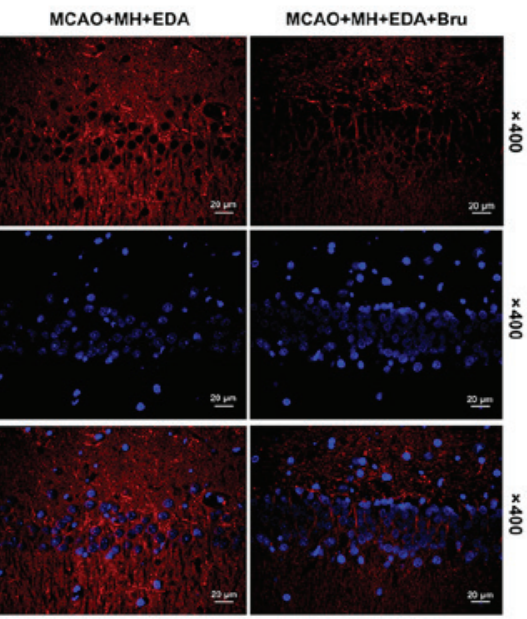

Figure 2. Effects of MH, EDA and Bru on spatial learning, memory and mitochondrial function in rats subjected to MCAO. (A and B) The escape latency and quadrant occupancy of the rats were evaluated by Barnes maze task on day 12 after MCAO; $\mathrm{n}=10$ mice per group. (C and D) Representative confocal microscopy images and statistical analysis of MitoRed fluorescence staining. Scale bar: $20 \mu \mathrm{m}$. Magnification, $\mathrm{x} 400$. ${ }^{* * *} \mathrm{P}<0.001 \mathrm{vs}$. sham. ${ }^{\# \# \#} \mathrm{P}<0.001 \mathrm{vs}$. MCAO. ${ }^{\wedge \wedge} \mathrm{P}<0.001$ vs. MCAO + MH + EDA. MCAO, middle cerebral artery occlusion; MH, mild hypothermia; EDA, edaravone; Bru, brusatol.

the $\mathrm{MCAO}+\mathrm{MH}+\mathrm{EDA}+\mathrm{Bru}$ group compared with those in the $\mathrm{MCAO}+\mathrm{MH}+\mathrm{EDA}$ group $(\mathrm{P}<0.001$, Fig. 3B-E). The primary hippocampal neurons were collected from the tissues of the rats in different groups, and the cell viability was measured by the CCK- 8 assay. The result demonstrated that MCAO reduced the viability of primary hippocampal neurons, which, however, was increased by $\mathrm{MH}$ and EDA treatment or their co-treatment. Moreover, it was observed that Bru injection suppressed the effects of $\mathrm{MH}$ and EDA $(\mathrm{P}<0.05$, Fig. 3F). Furthermore, the activity of cytochrome c oxidase and caspase-3 in the mitochondrial fraction was detected, and the data demonstrated that MCAO increased the levels of cytochrome c oxidase and caspase-3, while $\mathrm{MH}$ and EDA weakened the effect of MCAO; moreover, Bru treatment blocked the effects of $\mathrm{MH}$ and EDA on cytochrome c oxidase and caspase- 3 activities ( $\mathrm{P}<0.001$, Fig. $3 \mathrm{G}$ and $\mathrm{H}$ ).

Effects of $\mathrm{MH}, \mathrm{EDA}$ and Bru on the Nrf2/HO-1 pathway. The Nrf2 pathway is known to act protectively against oxidative stress. The expressions of cytoplasmic Nrf2 (cNrf2), nuclear Nrf2 (nNrf2), and those of its downstream genes HO-1 and NQO1 in rat ischemic hemisphere tissues were measured by western blotting. The data demonstrated that MCAO significantly increased the expression of cNrf2 $(\mathrm{P}<0.001)$ and slightly increased the expressions of Nrf2, HO-1 and NQO1 $(\mathrm{P}<0.01$ or $\mathrm{P}<0.001$, Fig. 4A-E). In addition, $\mathrm{MH}$ and EDA markedly increased the levels of HO-1, NQO1 and nNrf2, and simultaneously reduced the level of cNrf2, as compared with the MCAO group ( $\mathrm{P}<0.001$, Fig. 4A-E). The combined treatment with $\mathrm{MH}$ and EDA enhanced the effects caused by treatment with either
$\mathrm{MH}$ or EDA alone on the Nrf2/HO-1 pathway, whereas these effects were reversed by Bru treatment $(\mathrm{P}<0.05$ or $\mathrm{P}<0.001$, Fig. 4A-E).

\section{Discussion}

It has been demonstrated that MH used together with other drugs or treatments may enhance the protective effects of $\mathrm{MH}$ against cerebral I/R injury. For example, a recent research revealed that $\mathrm{MH}$ with bone mesenchymal stem cell transplantation improved the prognosis of rats with cerebral ischemia (19). Combined therapy with phenothiazines and $\mathrm{MH}$ also enhanced neuroprotection in ischemic rats through regulating the PI3K/Akt pathway (20). It has also been reported that the Notch inhibitor DAPT combined with MH exerted a synergistic effect on post-stroke seizures (21). It was reported that $\mathrm{MH}$ in combination with phenothiazine drugs achieved a better long-term motor performance after acute stroke (22). Moreover, $\mathrm{MH}$ used with hydrogen sulfide treatment suppressed apoptosis and pathological injury of hippocampi of rats with cerebral I/R injury (23). These findings indicated that $\mathrm{MH}$ combined with other treatments may be a promising therapy in the management of cerebral I/R injury. The present study demonstrated that the neurological deficit score was significantly reduced and neuronal density was increased in the CA1 region of the rat hippocampi, suggesting that EDA combined with $\mathrm{MH}$ exerted a synergistic effect against I/R injury.

Although neurological deficit score evaluation and Barnes maze task test were performed, it may be a limitation 
A

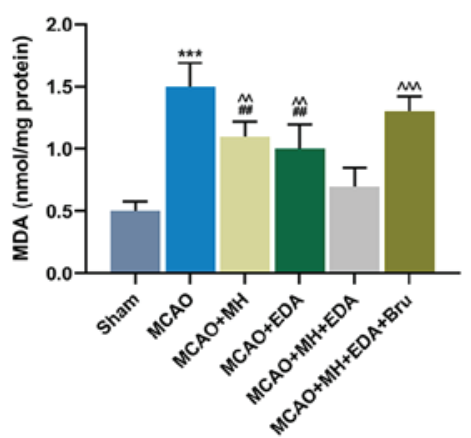

D

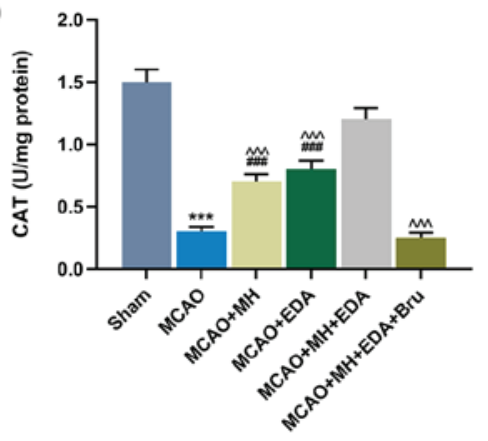

B

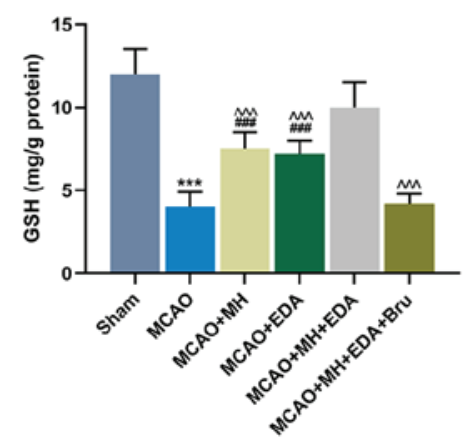

$\mathbf{E}$

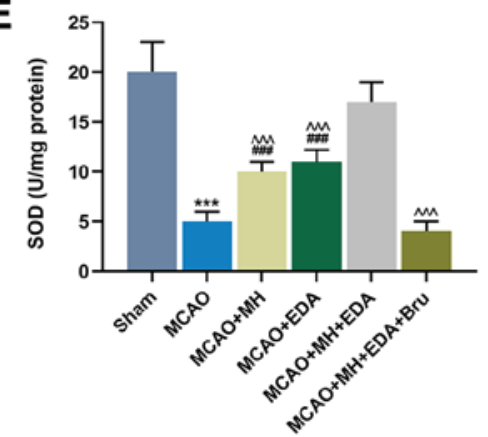

C

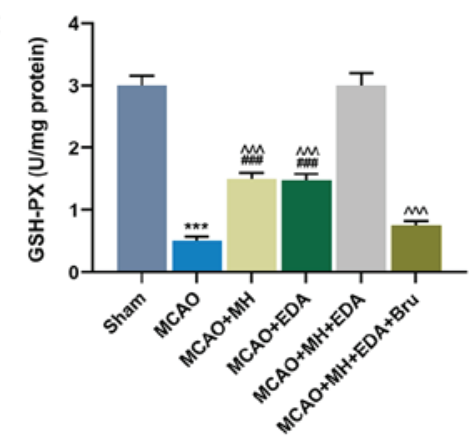

$\mathbf{F}$

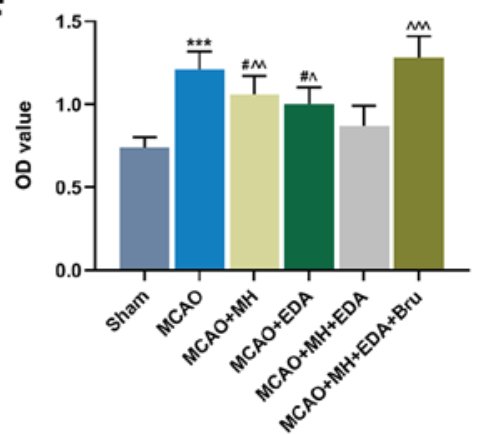

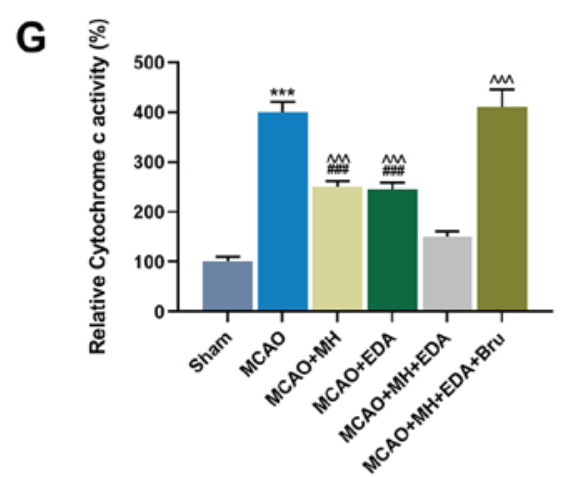

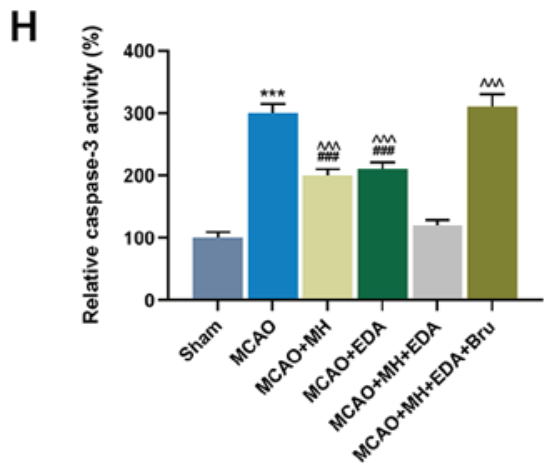

Figure 3. Effects of MH, EDA and Bru on the levels of oxidative stress indicators, neuronal viability, cytotoxicity and caspase-3 activity. (A-E) The levels of MDA, GSH, GSH-PX, CAT and SOD in rat ischemic hemispheres tissues were determined by colorimetric methods; $\mathrm{n}=10$ mice per group. (F) The viability of neurons from the CA1 region of hippocampi was determined by the MTT assay. ( $\mathrm{G}$ and $\mathrm{H}$ ) Cytochrome $\mathrm{c}$ and caspase-3 in the mitochondrial fractions from each group was detected by ELISA. ${ }^{* * *} \mathrm{P}<0.001$ vs. sham. ${ }^{\|} \mathrm{P}<0.1,{ }^{\# \#} \mathrm{P}<0.01$ and ${ }^{\# \# \#} \mathrm{P}<0.001$ vs. MCAO. ${ }^{\wedge} \mathrm{P}<0.1,{ }^{\wedge \wedge} \mathrm{P}<0.01$ and ${ }^{\wedge \wedge} \mathrm{P}<0.001 \mathrm{vs}$. MCAO + MH $+\mathrm{EDA}$. MCAO, middle cerebral artery occlusion; MH, mild hypothermia; EDA, edaravone; Bru, brusatol; MDA, malondialdehyde; GSH, glutathione; GSH-PX, glutathione peroxidase; CAT, catalase; SOD, superoxide dismutase.

that the TTC dying method was not used to assess the brain infarct area. The hippocampus is responsible for spatial learning and memory (24), which may be disturbed when the hippocampus sustains cerebral I/R injury (25). Therefore, the Barnes Maze task that helps evaluate spatial learning and memory ability was performed in the present study, and it was observed that EDA combined with $\mathrm{MH}$ could improve the spatial learning and memory ability of rats with cerebral I/R injury. MitoRed fluorescence staining was used to evaluate MMP. Under normal physiological conditions, mitochondria with high MMP can be marked by Mito-Tracker Red CMXRos, while MMP is reduced in mitochondrial dysfunction and therefore cannot be marked by Mito-Tracker Red CMXRos under pathological conditions or when cell apoptosis occurs. The present study demonstrated that mitochondrial dysfunction in rat hippocampi was induced by MCAO, but was significantly restored by the combined therapy of EDA with $\mathrm{MH}$.
The CAT, SOD, GSH and GSH-PX enzymes are involved in the antioxidant defense system against cell oxidative stress (26), and MDA is indicative of IR-induced mitochondrial oxidative damage (27). In the present study, it was observed that MDA was increased, but CAT, SOD, GSH and GSH-PX were reduced in rats subjected to MCAO, suggesting that mitochondrial oxidative damage was induced by cerebral I/R injury. Importantly, restoration of dysregulated MDA, CAT, SOD, GSH and GSH-PX levels was detected in rats treated by MCAO and in those treated by combined EDA and MH. Thus, it may be inferred that EDA combined with MH alleviated the mitochondrial dysfunction induced by cerebral I/R.

Neurogenesis may be enhanced under pathological conditions, including cerebral ischemic injury, and it promotes repair of brain injury after stroke. It was demonstrated that neurogenesis can be suppressed by EDA in rats with cerebral I/R injury (28). However, it has also been shown that EDA 


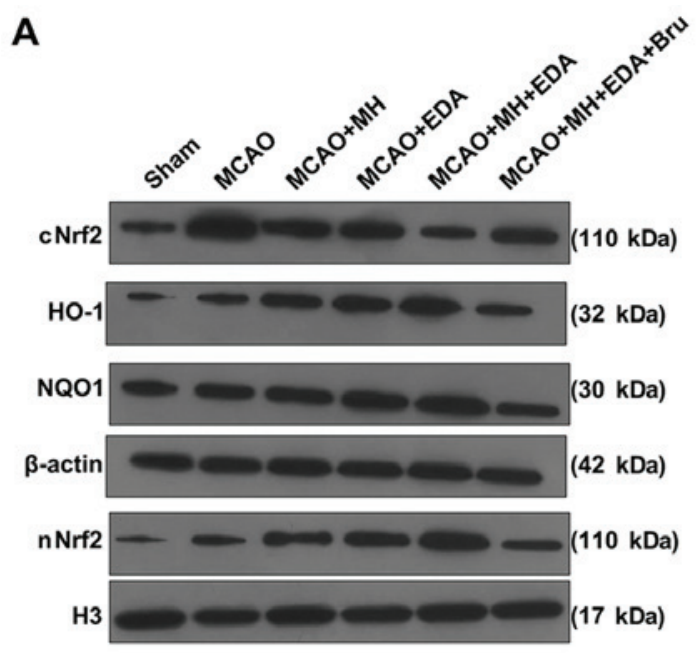

B

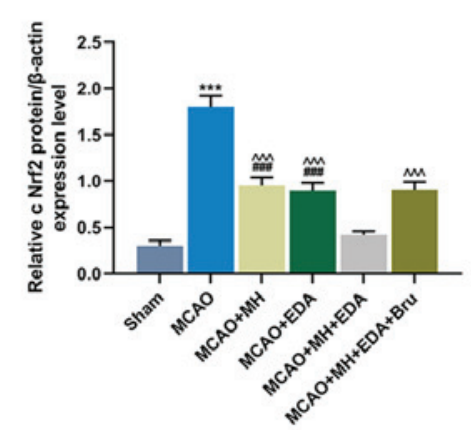

D

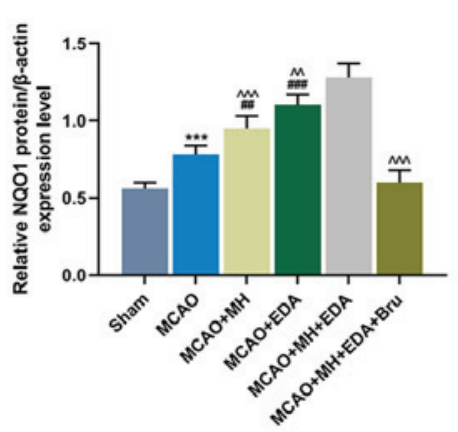

C

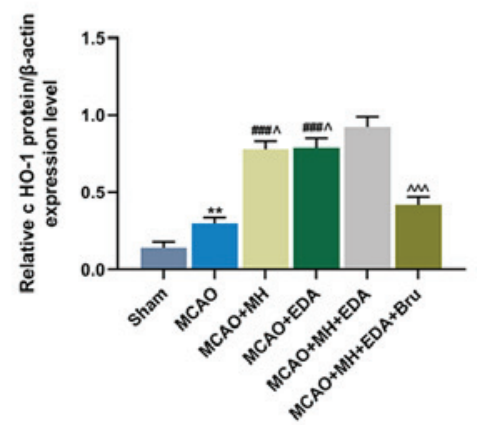

$\mathbf{E}$

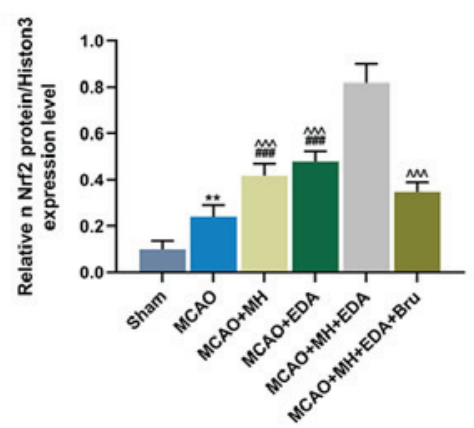

Figure 4. Effects of MH, EDA and Bru on the activation of the Nrf2/HO-1 pathway. (A-E) Western blot analyses of expressions of cNrf2, HO-1, NQO1, $\beta$-actin, nNrf2 and histone $\mathrm{H} 3$ in rat ischemic hemisphere tissues; $\mathrm{n}=10$ mice per group. ${ }^{* * *} \mathrm{P}<0.01$ and ${ }^{* * * *} \mathrm{P}<0.001$ vs. sham. ${ }^{\# \#} \mathrm{P}<0.001$ and ${ }^{\# \# \#} \mathrm{P}<0.001$ vs. MCAO. ${ }^{\wedge} \mathrm{P}<0.1$, ${ }^{\wedge} \mathrm{P}<0.01$ and ${ }^{\wedge \wedge} \mathrm{P}<0.001$ vs. MCAO + MH + EDA. MCAO, middle cerebral artery occlusion; MH, mild hypothermia; EDA, edaravone; Bru, brusatol; Nrf2, nuclear factor erythroid 2-related factor 2; cNrf2, cytoplasmic Nrf2; nNrf2, nuclear Nrf2; HO-1, heme oxygenase-1; NQO1, NAD(P)H quinone oxidoreductase.

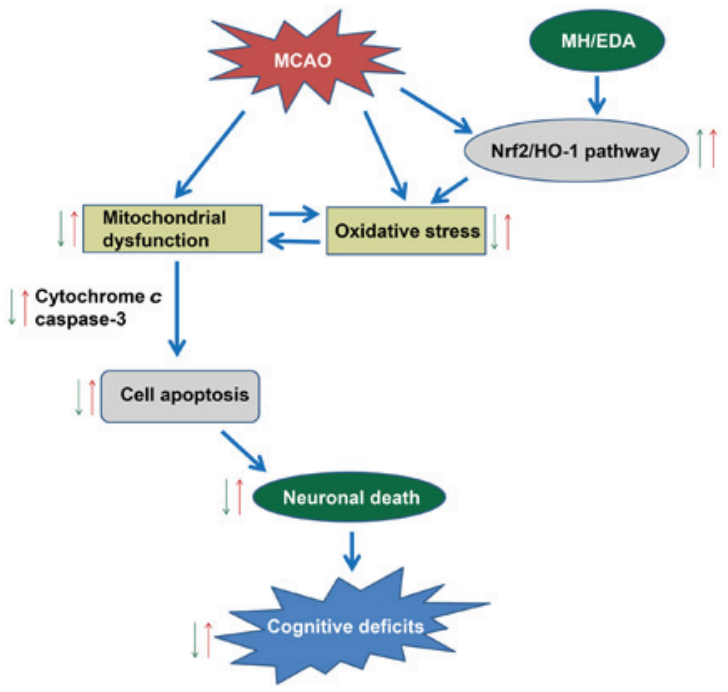

Figure 5. Working model of the protective effects of MH/EDA on MCAO-induced cognitive deficits (red arrow, effect of MCAO; green arrow, effect of MH/EDA). MCAO caused cognitive deficits through promoting mitochondrial dysfunction and increasing the oxidative stress level. The Nrf2/HO-1 pathway was activated as protection against oxidative stress. The promoting effect on mitochondrial dysfunction and oxidative stress induced an increase in cytochrome $\mathrm{c}$ and caspase-3 levels, thereby inducing cell apoptosis and neuronal death. Combined therapy with MH and EDA play a protective role through promoting the activation of the Nrf2/HO-1 pathway, thereby reducing the oxidative stress and mitochondrial dysfunction. MCAO, middle cerebral artery occlusion; $\mathrm{MH}$, mild hypothermia; EDA, edaravone; Nrf2, nuclear factor erythroid 2-related factor 2; HO-1, heme oxygenase-1.

protects hippocampal neurons pre- and post-treatment and enhances neurogenesis after global cerebral ischemia (29).
In the present research, the viability of neurons separated from the CA1 region of the hippocampus was increased by MCAO, but was suppressed by MH and EDA. In addition, the increased activities of cytochrome $c$ and caspase- 3 were partially reversed by the combination of $\mathrm{MH}$ with EDA in rats with cerebral I/R. I/R induces dysfunction of mitochondria and reduces cytochrome $\mathrm{c}$ oxidase activity in rat hippocampal neurons $(30,31)$. Increased activity of cytochrome $\mathrm{c}$ increases oxidative stress and cell apoptosis. Caspase- 3 is considered to be a cell apoptosis marker; thus, it was inferred that MCAO may promote neurogenesis and increase the levels of apoptosis-associated factors, whereas MH and EDA may inhibit this process. It should be noted that the effect of the combined therapy with $\mathrm{MH}$ and EDA on cell apoptosis require further investigation.

$\mathrm{Nrf} 2$ is a regulator of endogenous antioxidant defense and may be a potential target for oxidative stress in stroke (32). Nrf2 induces HO-lexpression and release of GSH to resist oxidative stress (33). It was reported that BRCA1 protects neurons from cerebral I/R injury through activating the Nrf2-associated antioxidant pathways (34). It has been reported that EDA enhances the oxidant defense capacity in brain tissue through promoting Nrf2/HO-1 signaling (35). MH was also reported to protect cerebral cortex and hippocampus neurons from cerebral I/R injury through the Nrf2/ARE pathway (36). In the present study, by injecting the Nrf2 pathway inhibitor Bru into rats treated by MH combined with EDA, it was observed that the protective effect of MH combined with EDA is linked to the Nrf2 pathway. A previous study demonstrated that nuclear-translocated Nrf2 binds to ARE and p300/CBP to promote the transcription 
of downstream target genes, such as HO-1 (37). The present study revealed that nuclear location of Nrf2 was increased and the expression of the Nrf2 downstream genes NQO1 and HO-1 was upregulated in the treated by $\mathrm{MH}$ combined with EDA.

In conclusion, a rat cerebral ischemia model was constructed in the present study, and demonstrated that combined treatment with $\mathrm{MH}$ and EDA improved rat neurological function and increased neuronal survival. In addition, the Nrf2 pathway inhibitor, Bru, could block the protective effect of $\mathrm{MH}$ and EDA. These findings indicated that $\mathrm{MH}$ combined with EDA exert a synergistic effect against cerebral I/R injury through the Nrf2/HO-1 pathway. However, a limitation of the present study was that there was no detection of more indicators of oxidative stress, and further research on these findings is required.

\section{Acknowledgements}

Not applicable.

\section{Funding}

The present study was supported by the Hainan Provincial Health and Family Planning Research Project (grant no. 18A200002), the Hainan Provincial Higher Education Research Project (grant no. Hnky2018-50) and the National Natural Science Foundation of China (grant no. 81660270).

\section{Availability of data and materials}

The data sets generated and/or analyzed during the present study are available from the corresponding author on reasonable request.

\section{Authors' contributions}

Substantial contributions to conception and design: HY and $\mathrm{ZW}$; data acquisition, data analysis and interpretation: XW, CG, RL, FK and MD; drafting the article and critically revising it for important intellectual content: $\mathrm{HY}$ and $\mathrm{ZW}$. All the authors have read and approved the final version to be published and agree to be accountable for all aspects of the work in ensuring that questions related to the accuracy or integrity of the work are appropriately investigated and resolved.

\section{Ethics approval and consent to participate}

All the animal experiments were approved by the Institutional Animal Ethics Committee of Hainan Medical University (no. C2017051922A).

\section{Patient consent for publication}

Not applicable.

\section{Competing interests}

All the authors declare that they have no competing interests.

\section{References}

1. Alkhachroum Am, Miller B, Chami T, Tatsuoka C and Sila C: A troponin study on patients with ischemic stroke, intracerebral hemorrhage and subarachnoid hemorrhage: Type II myocardial infarction is significantly associated with stroke severity, discharge disposition and mortality. J Clin Neurosci 64: 83-88, 2019.

2. Feigin VL, Mensah GA, Norrving B, Murray CJ and Roth GA; GBD 2013 Stroke Panel Experts Group: Atlas of the Global Burden of Stroke (1990-2013): The GBD 2013 Study. Neuroepidemiology 45: 230-236, 2015.

3. Vos T, Abajobir AA, Abate KH, Abbafati C, Abbas KM, Abd-Allah F, Abdulkader RS, Abdulle AM, Abebo TA, Abera SF, et al; GBD 2016 Disease and Injury Incidence and Prevalence Collaborators: Global, regional, and national incidence, prevalence, and years lived with disability for 328 diseases and injuries for 195 countries, 1990-2016: A systematic analysis for the Global Burden of Disease Study 2016. Lancet 390: 1211-1259, 2017.

4. Cheung RT: The utility of melatonin in reducing cerebral damage resulting from ischemia and reperfusion. J Pineal Res 34: 153-160, 2003.

5. Zerna C, Thomalla G, Campbell BCV, Rha JH and Hill MD: Current practice and future directions in the diagnosis and acute treatment of ischaemic stroke. Lancet 392: 1247-1256, 2018.

6. Leng T, Shi Y, Xiong ZG and Sun D: Proton-sensitive cation channels and ion exchangers in ischemic brain injury: New therapeutic targets for stroke? Prog Neurobiol 115: 189-209, 2014.

7. Tang XN, Liu L, Koike MA and Yenari MA: Mild hypothermia reduces tissue plasminogen activator-related hemorrhage and blood brain barrier disruption after experimental stroke. Ther Hypothermia Temp Manag 3: 74-83, 2013.

8. Tu Y, Guo C, Song F, Huo Y, Geng Y, Guo M, Bao H, Wu X and Fan W: Mild hypothermia alleviates diabetes aggravated cerebral ischemic injury via activating autophagy and inhibiting pyroptosis. Brain Res Bull 150: 1-12, 2019.

9. Yang GS, Zhou XY, An XF, Liu XJ, Żhang YJ and Yu D: Mild hypothermia inhibits the Notch 3 and Notch 4 activation and seizure after stroke in the rat model. Pathol Res Pract 214: 1008-1016, 2018.

10. Liu LQ, Liu XR, Zhao JY, Yan F, Wang RL, Wen SH, Wang L, Luo YM and Ji XM: Brain-selective mild hypothermia promotes long-term white matter integrity after ischemic stroke in mice. CNS Neurosci Ther 24: 1275-1285, 2018.

11. Yuan $\mathrm{Y}$, Zha H, Rangarajan $\mathrm{P}$, Ling EA and $\mathrm{Wu} \mathrm{C}$ : Anti-inflammatory effects of Edaravone and Scutellarin in activated microglia in experimentally induced ischemia injury in rats and in BV-2 microglia. BMC Neurosci 15: 125, 2014.

12. Li L, Yang R, Li P, Lu H, Hao J, Li L, Tucker D and Zhang Q: Combination Treatment with Methylene Blue and Hypothermia in Global Cerebral Ischemia. Mol Neurobiol 55: 2042-2055, 2018.

13. Shibuta S, Varathan S, Kamibayashi T and Mashimo T: Small temperature variations alter edaravone-induced neuroprotection of cortical cultures exposed to prolonged hypoxic episodes. $\mathrm{Br} \mathrm{J}$ Anaesth 104: 52-58, 2010.

14. Dodson M, de la Vega MR, Cholanians AB, Schmidlin CJ, Chapman E and Zhang DD: Modulating NRF2 in Disease: Timing Is Everything. Annu Rev Pharmacol Toxicol 59: 555-575, 2019.

15. Shou L, Bei Y, Song Y, Wang L, Ai L, Yan Q and He W: Nrf2 mediates the protective effect of edaravone after chlorpyrifos-induced nervous system toxicity. Environ Toxicol 34: 626-633, 2019.

16. Li C, Mo Z, Lei J, Li H, Fu R, Huang Y, Luo S and Zhang L: Edaravone attenuates neuronal apoptosis in hypoxic-ischemic brain damage rat model via suppression of TRAIL signaling pathway. Int J Biochem Cell Biol 99: 169-177, 2018.

17. Cen J, Zhao N, Huang WW, Liu L, Xie YY, Gan Y, Wang CJ and Ji BS: Polyamine analogue QMA attenuated ischemic injury in MCAO rats via ERK and Akt activated Nrf2/HO-1 signaling pathway. Eur J Pharmacol 844: 165-174, 2019.

18. Longa EZ, Weinstein PR, Carlson S and Cummins R: Reversible middle cerebral artery occlusion without craniectomy in rats. Stroke 20: 84-91, 1989.

19. Bi M, Wang J, Zhang Y, Li L, Wang L, Yao R, Duan S, Tong S and Li J: Bone mesenchymal stem cells transplantation combined with mild hypothermia improves the prognosis of cerebral ischemia in rats. PLoS One 13: e0197405, 2018. 
20. An H, Duan Y, Wu D, Yip J, Elmadhoun O, Wright JC, Shi W, Liu K, He X, Shi J, et al: Phenothiazines Enhance Mild Hypothermia-induced Neuroprotection via PI3K/Akt Regulation in Experimental Stroke. Sci Rep 7: 7469, 2017.

21. Yang GS, Zhou XY, An XF, Liu XJ,Zhang YJ and Yu D: Synergistic effect of mild hypothermia and the Notch inhibitor DAPT against post stroke seizures. Biomed Pharmacother 96: 675-684, 2017.

22. Liu S, Geng X, Forreider B, Xiao Y, Kong Q, Ding Y and Ji X: Enhanced beneficial effects of mild hypothermia by phenothiazine drugs in stroke therapy. Neurol Res 37: 454-460, 2015.

23. Dai HB, Xu MM, Lv J, Ji XJ,Zhu SH, Ma RM, Miao XL and Duan ML: Mild hypothermia combined with hydrogen sulfide treatment during resuscitation reduces hippocampal neuron apoptosis via NR2A, NR2B, and PI3K-Akt signaling in a rat model of cerebral ischemia-reperfusion injury. Mol Neurobiol 53: 4865-4873, 2016.

24. Jeffery KJ: The Hippocampus: From Memory, to Map, to Memory Map. Trends Neurosci 41: 64-66, 2018.

25. Xu F, Zhang G, Yin J, Zhang Q, Ge MY, Peng L, Wang S and Li Y: Fluoxetine mitigating late-stage cognition and neurobehavior impairment induced by cerebral ischemia reperfusion injury through inhibiting ERS-mediated neurons apoptosis in the hippocampus. Behav Brain Res 370: 111952, 2019.

26. Das KK, Gupta AD, Dhundasi SA, Patil AM, Das SN and Ambekar JG: Protective role of L-ascorbic acid on antioxidant defense system in erythrocytes of albino rats exposed to nickel sulfate. Biometals 20: 177-184, 2007.

27. Yang Y, Duan W, Jin Z, Yi W, Yan J, Zhang S, Wang N, Liang Z, Li Y, Chen W, et al: JAK2/STAT3 activation by melatonin attenuates the mitochondrial oxidative damage induced by myocardial ischemia/reperfusion injury. J Pineal Res 55: 275-286, 2013.

28. Zhang P, Li W, Li L, Wang N, Li X, Gao M,Zheng J, Lei S, Chen X, $\mathrm{Lu} \mathrm{H}$, et al: Treatment with edaravone attenuates ischemic brain injury and inhibits neurogenesis in the subventricular zone of adult rats after focal cerebral ischemia and reperfusion injury. Neuroscience 201: 297-306, 2012.

29. Lei S, Zhang P, Li W, Gao M, He X, Zheng J, Li X, Wang X, Wang N, Zhang J, et al: Pre- and posttreatment with edaravone protects CA1 hippocampus and enhances neurogenesis in the subgranular zone of dentate gyrus after transient global cerebral ischemia in rats. ASN Neuro 6: 1759091414558417, 2014
30. Racay P, Tatarková Z, Drgová A, Kaplan P and Dobrota D: Ischemia-reperfusion induces inhibition of mitochondrial protein synthesis and cytochrome c oxidase activity in rat hippocampus. Physiol Res 58: 127-138, 2009.

31. Zhong S, Li Z, Huan L and Chen BY: Neurochemical mechanism of electroacupuncture: Anti-injury effect on cerebral function after focal cerebral ischemia in rats. Evid Based Complement Alternat Med 6: 51-56, 2009.

32. Zhang R, Xu M, Wang Y, Xie F, Zhang G and Qin X: Nrf2-a promising therapeutic target for defensing against oxidative stress in stroke. Mol Neurobiol 54: 6006-6017, 2017.

33. Wu L, Li HH, Wu Q, Miao S, Liu ZJ, Wu P adn Ye DY: Lipoxin A4 activates Nrf2 pathway and ameliorates cell damage in cultured cortical astrocytes exposed to oxygen-glucose deprivation/reperfusion insults. J Mol Neurosci 56: 848-857, 2015.

34. Xu P, Liu Q, Xie Y, Shi X, Li Y, Peng M, Guo H, Sun R, Li J, Hong Y, et al: Breast cancer susceptibility protein 1 (BRCA1) rescues neurons from cerebral ischemia/reperfusion injury through NRF2-mediated antioxidant pathway. Redox Biol 18: 158-172, 2018.

35. Li Z, Yulei J, Yaqing J, Jinmin Z, Xinyong L, Jing G and Min L: Protective effects of tetramethylpyrazine analogue Z-11 on cerebral ischemia reperfusion injury. Eur J Pharmacol 844: 156-164, 2019.

36. Xia D and Zhang H: Effects of mild hypothermia on expression of NF-E2-related factor 2 and heme-oxygenase-1 in cerebral cortex and hippocampus after cardiopulmonary resuscitation in rats. Iran J Basic Med Sci 20: 1002-1008, 2017.

37. Kim SW, Lee HK, Shin JH and Lee JK: Up-down regulation of $\mathrm{HO}-1$ and iNOS gene expressions by ethyl pyruvate via recruiting p300 to Nrf2 and depriving It from p65. Free Radic Biol Med 65: 468-476, 2013.

(†) $\Theta$ This work is licensed under a Creative Commons Attribution-NonCommercial-NoDerivatives 4.0 International (CC BY-NC-ND 4.0) License. 